\title{
EFFICIENT HIERARCHICAL ROUTING PROTOCOL IN SENSOR NETWORKS
}

\author{
S.Karthikeyan ${ }^{1}$ and S.Jayashri ${ }^{2}$ \\ ${ }^{1}$ Research scholar, Sathyabma University, chennai, India \\ karthijoyl@gmail.com \\ ${ }^{2}$ Principal, Adhiparasakthi Engineering college, Melmaruvathur, Tamil Nadu, India \\ jayaravi2010@gmail.com
}

\begin{abstract}
Wireless sensor network (WSN) is the network of hundreds and thousands of micro-sensor nodes, connecting each other by a wireless medium.WSN provide reliable sensing of the environment, detecting and reporting events to the sink. One of the most important constraints of WSN is energy consumption. Since the micro sensors are small in dimension, batteries are necessary to produce power to the network. In this paper, we have proposed an algorithm for hierarchy based protocols of wireless sensor networks, which consist of two groups of sensor nodes in a single cluster node. Each cluster consists of a three cluster head. The event driven data sensing mechanism is used in this paper and this sensed data is transmitted to the master section head. The gathered data is transmitted to the sink via mobile agent. Hence efficient way of data transmission is possible with larger group of nodes. In this approach of using hierarchy based protocols; the lifetime of the sensor network is increased. This paper proposes an innovative approach of cluster head election. The results are compared with LEACH protocol and proved to be energy efficient.
\end{abstract}

\section{KEYWORDS}

Sensor Nodes, lifetime, event driven, master section head, Mobile Agent.

\section{INTRODUCTION}

With the advancements in Wireless communication The design and implementation of WSNs have become a sizzling and important area of research. The micro-sensors along with compact and portable computing devices have made the distributed sensing of greater importance. It enables the applications to connect the physical world to the virtual world due to the latent of sensor networks. To obtain the information about the physical environment was in fact difficult or almost impossible to obtain in more conventional ways. But by introducing the sensor networks with tiny sensor nodes, the whole picture turned upside-down, turned impossible possible. As micro-fabrication technology will become advanced in the future it will ultimately allow the increase deployments of wireless sensor networks and cost of manufacturing sensor nodes to fall, where the networks are growing rapidly to large number of nodes, for e.g., thousands[1]. When compared to data processing, the data transmission consumes more energy in WSN. The data aggregation will balance the energy consumption of each node, so that the network's lifetime is increased [2],[3].The major advantages of WSN over conventional networks are accuracy, low cost, greater coverage area and reliability. WSNs are immobile, comprises large number of tiny micro sensors, which is more than the nodes in traditional networks. The WSN perform a specific data gathering and transmission by deploying large number of sensor nodes randomly. It has a base station called as sink, which receives the transmitted data. For such large scale wireless

DOI:10.5121/ijassn.2013.3202 
sensor networks large number of potential applications exist in a variety of fields like medical monitoring, environmental monitoring, surveillance, security, military operations and industrial machine monitoring. For better view and understanding as to why traditional protocols are not best suited for these types of sensor network applications, the unique features of sensor networks and the performance metrics with which the protocols for the sensor networks must be evaluated will be categorized in the remainder of this section. To achieve the specific performance requirements of these networks one of the popular approaches used is Clustering. After clustering, the cluster head collects and gathers the sensed data and transmits to the sink. The cluster head election is rotated to share the burden of the head and balance the energy consumption [4]. There is always a limitation on the cost and size of the network, because the power of the nodes depends on the actual power embedded in the nodes [5].

\subsection{Features of WSN}

Most of the ad hoc network features are shared by WSN. Thus protocol design which is used for sensing networks must include for the properties of ad-hoc networks [6]. Some of the features of Sensor networks are given here. The communication is unreliable due to the usage of wireless medium. The lifetime of the network is affected by the limited energy supplies of the nodes in the network. However several unique features exist in wireless sensor networks that don't even exist in general ad hoc networks. These features will require modification of designs for ad hoc network as they have to face new challenges. The ad hoc network has lesser size, whereas the sensor networks have thousands of nodes in it. The sensor nodes are typically immobile, which clearly means that the mechanism used in traditional ad-hoc network protocols to deal with mobility may be unnecessary and may also look overweight. Unexpected node failure is more, since they are deployed in harsh environmental conditions. The nodes used in WSN are very tiny micro sensors, which are much smaller than nodes used in traditional ad hoc networks (e.g., PDAs, laptop computers), These nodes consist of smaller batteries which leads to shorter life time, less computational power and also less memory. Additional services such as location information may also be required in wireless sensor networks. In the traditional ad hoc networks nodes compete for resources such as bandwidth whereas nodes in a sensor network are expected to behave more cooperatively because they try to accomplish a similar universal goal, which is typically related to maintaining an application-level quality of service (QOS), or fidelity. Communication is actually data-centric rather than address-centric, which means that depending on the description of the data, routed data may be aggregated or compressed or prioritized or dropped. Communication in the sensor networks typically take place in the form of very short packets, which means that the relative overhead imposed at different network layers have become much more important.

\subsection{Challenges in WSN Design}

WSN design is motivated and influenced by one or more of the following technical challenge: Mostly the WSNs are randomly deployed, where the large no of nodes are distributed densely across large region. The dense deployment of sensor nodes leads to high correlation of data in the neighbourhood that are sensed by the nodes.

WSN has four design constraints, which are bandwidth, memory energy and consumption. Because of its small size micro sensors could only be attached with bounded battery energy supply. The WSN batteries are non rechargeable and/or irreplaceable. The memory limitation allows them to perform with restricted computational functionality. The connectivity and topology of WSNs may frequently vary due to the unreliability of the individual wireless microsensors. Sensor nodes incur more errors since it uses wireless medium. The communication environment is mostly noisy and can cause severe signal distortion. WSN is used for wide range 
of tasks, such as target detection and tracking, environment monitoring, remote sensing, military surveillance, etc., Requirements for the different applications may vary significantly. Privacy and safety should be essential considerations in the design of WSNs because many of them are used for surveillance or military purposes. The accuracy of data reported to what is actually occurring in the environment represents the quality of WSN. The way to measure accuracy is the amount of data. Latency is another aspect of accuracy. Data collected by WSNs are typically time sensitive, e.g., early warning of fires. It is therefore important to receive the data at the destination/control centre in a timely manner. With long latency may be outdated and lead to wrong reactions [6] due to processing or communication data.

\section{TAXONOMY FOR ROUTING PROTOCOLS}

Classification of routing protocols for network structure is

\subsection{Flat-based routing}

In the flat based routing, feature of unique global identifier cannot be provided for each node because of large number of nodes. Equal roles are assigned to deployed nodes in the networks. Data-centric routing, where queries are given by the destination node to the particular region of the nodes. Data is delivered after a delay period from the region of that particular sensed node. Data request is based on queries, properties of the data is specifically necessary for this attribute based naming. For e.g., SPIN, Rumour routing, DD and Gradient based routing (GBR).

\subsection{Hierarchical-based routing (Cluster-based routing)}

Sensor nodes can play different roles in the networks and the protocol is based on the cluster creation. Cluster creation and specific task assignment to cluster head contributes the overall system energy efficiency, scalability, and lifetime of the network. Hierarchical routing performs aggregation and data fusion which in result decreases the number of information message transmitted to the destination node. The hierarchical based routing gives an energy efficient way of reducing the energy consumption in the cluster.

In addition to this, sensor nodes with different characteristics of data transmission are performed. For e.g., Power-Efficient Gathering in Sensor Information Systems (PEGASIS), Low Energy Adaptive Clustering Hierarchy (LEACH), Threshold-Sensitive Energy Efficient Protocols (TEEN).

\subsection{Location-based routing}

Location based addressing of nodes is formed in this routing protocol. Distance between the node A and the neighbour are estimated by calculating the signal strength or by GPS receivers. For e.g., Geographic Adaptive Fidelity (GAF), Geographic and Energy Aware Routing (GEAR).

\section{HIERARCHY BASED PROTOCOL}

\subsection{LEACH}

LEACH (Low Energy Adaptive Clustering Hierarchy) is cluster-based protocol. It has distributed cluster formation. In LEACH, the cluster head selection is random among the group of distributed nodes. The role of cluster head is rotated to evenly distribute energy of the sensor nodes in the networks. The operation of collecting the data from the sensor nodes and transmit it to the base 
station is done by LEACH. Using LEACH the cluster head $(\mathrm{CH})$ compress the information packet received from different nodes within the same cluster and the aggregated data packet are transmitted to the base station which reduces the size of the information to be transmitted to the base station. The cluster head rotates, to avoid energy dissipation due to data transfer to the base station. This leads to balanced energy consumption of the network, which in turn increases the lifetime of the network [7]. This protocol uses the TDMA/CDMA medium access control (MAC) which reduces collision in the inter-cluster and Intra-cluster data transmission. Centralized data collection is done in a periodical basis. In this protocol, there happens constant monitoring of the sensor nodes in the networks. Periodically data transmission will drain the limited energy of the sensor nodes in the network. Since user does not need all the data instantaneously. Unnecessary data transmission is avoided to save energy in sensor nodes. In addition to improvement in energy density, energy consumption is reduced. Rotation of the cluster head is randomized after a period of time. So that energy distributed in the sensor nodes will be even after a certain period of time. LEACH is operated in two phases, the setup phase and the steady state phase. In the setup phase, organized clusters and Cluster head selection takes place. In the steady state phase, the actual data is transferred to the base station. The steady state phase duration is longer than the duration of the setup phase to minimize the overhead. A predetermined fraction of nodes, $p$, elect themselves as CHs as follow in the setup phases. Sensor node with the random number (r) is chosen between 0 and1. If the Compared random number is less than the threshold value, $T(n)$, for the current round then the node becomes a cluster-head. The threshold value is calculated based on the desired percentage to become a cluster head, current round and nodes that has not appeared as a cluster-head in the last $(1 / \mathrm{p})$ rounds, which is denoted by $\mathrm{G}$.

$\mathrm{T}(\mathrm{n})=\left\{\begin{array}{l}\mathrm{P}^{\prime} /\left(1-\mathrm{p} *\left(\mathrm{r} \bmod \left(\frac{1}{\mathrm{p}}\right)\right) \quad \text { If } \mathrm{n} \in \mathrm{G}\right. \\ 0\end{array}\right.$

Where $\mathrm{G}$ is the group of nodes involved in the cluster head selection process. After $\mathrm{CH}$ selection, $\mathrm{CH}$ broadcasts an advertisement message as a new cluster-heads to the group of the sensor nodes in the network [8]. Non-cluster head receives the advertisement message and from there it decides which cluster they want to join. The advertisement the nodes are grouped based on the strength of the advertisement. Member allocation takes place so that the non-cluster nodes inform the $\mathrm{CH}$ that they will be a part of this cluster. Messages from different nodes are received by the $\mathrm{CH}$ that it would like to be a part of the cluster, based on the number of nodes in the cluster. TDMA schedule is created by the cluster-head nodes and each node is assigned with a time slot by which it can transmit.

This schedule is being broadcasted to each and every node present in the cluster. Data is sensed and transmitted to the cluster heads by the sensor nodes during the steady state phase. The cluster head node receives all the data and then it aggregates all the data before sending the data to the base station. After a definite period of time, which will be called as priori the network will go back to the setup phase and then it will go for another round of selecting new $\mathrm{CH}$. Cluster will use different CDMA codes to communicate. This is done to reduce interference from nodes which belong to other clusters. LEACH increases the network lifetime, but there will be a number of issues for the assumptions used for this protocol.

The distance between two nodes $i$ and $j$ are calculated by using the equation 2 . This distance is calculated for the neighbour node selection [8].

Distance $\left.\left.(i, j)=\sqrt{\left(\left(X_{1}\right.\right.}-X_{2}\right)^{\wedge} 2+\left(Y_{1}-Y_{2}\right)^{\wedge} 2\right)$ 
Received signal strength Indicator is calculated by using the equation 3.

$\mathrm{RSSI}=-\left(10 n \log _{10} d+\mathrm{A}\right) \mathrm{A}$

Where $\mathrm{n}=$ signal propagation strength, $\mathrm{d}=$ Distance from the sender, $\mathrm{A}=$ Received signal strength indicator at 1 meter distance. Based on distance (d), the $n$ value decreases and value A increases.

The main work of LEACH is to watch whether all nodes can transmit with sufficient power to reach the $\mathrm{BS}$ if it is required to. With this each node will have computational power for supporting different MAC protocols. But networks are not allowed to be deployed in large regions. It predicts that nodes must always consist of data to send $\&$ the nodes which are located nearby will have correlated data. The number of predetermined $\mathrm{CH}(\mathrm{p})$ need not be necessarily distributed throughout the network uniformly. There could be a probability that the selected CHs could be concentrated in any one part of the network [9]. Therefore there is a possibility that that some nodes will not have any $\mathrm{CH}$ 's in their vicinity. Moreover, the concept of dynamic clustering brings an additional concept for eg., head changes, advertisements etc., which reduces the gain in energy consumption. At last the protocols assure that all the nodes must begin with the same amount of energy capacity in each of the selection round. It is assumed that $\mathrm{CH}$ will consume almost the same amount of energy for each node. The protocol should extend to account for non uniform energy nodes, i.e., by using energy-based threshold.

\subsection{TEEN and APTEEN}

Two hierarchical routing protocols are proposed for time-critical applications namely TEEN (Threshold-sensitive Energy Efficient sensor Network) and APTEEN (Adaptive Periodic Threshold-sensitive Energy Efficient sensor Network protocol). They are energy efficient hierarchy based routing protocol. They make use of a data centric mechanism. The medium is sensed by the sensor nodes in the case of teens, whereas data transmission is done very rarely. Within every cluster, one of the nodes is selected as cluster head. A cluster head is used to send its members a hard threshold which results as the threshold value of the sensed attribute, that is the minimum value of the sensed attribute to force the node to initiate transmission and a soft threshold, which is actually a differential change in the value of sensed attribute that is used to trigger the node to switch on its transmitter and it transmit. Now the hard threshold gets activated and it tries to reduce the number of transmission by allowing the nodes to transmit only sensed attribute[10] comes under the range of interest. The soft threshold in result reduces the number of transmission otherwise it may happen when there is little or no change in sensed attribute. When the value of soft threshold is small it gives a more perfect picture of the network with increased energy consumption. Each cluster head gathers and aggregates the data received and transmits it to the base station. In such cases, the user is able to control the trade-off between energy efficiency and data accuracy. When cluster-heads are needed to be changed, new set of values are broadcasted for the above parameters. TEEN is designed in such a way that it can react to the sudden alterations of the sensed element [11]. The ultimate drawback of this scheme is that, if the threshold is not received, the nodes can never communicate and the user will not be able to get any sort of data from the network. The nodes will be sensing their environment continuously. For the first time when a parameter from attribute set reaches its hard threshold value, the node will switch its transmitter to on state and then it sends the sensed data. The sensed value is store in an internal variable known as sensed value (sv). The nodes are able to transmit data in the period of current cluster only if the following conditions are satisfied. The conditions are:- 1) The current of the sensed attribute must be greater then the hard threshold. 2) The current value of the sensed attribute must differ from SV by an amount equal to or greater than the soft threshold. 
The important features of TEEN include its suitability for the time critical sensing applications. Also, as message transmission will consume more energy than data sensing, the amount of energy consumption in this scheme is less than the proactive networks. The soft threshold can also be varied. For every cluster time change, a set of fresh parameters are broadcasted and so, the user can change them as it is required. APTEEN is an extension of TEEN, which possess hybrid protocol enabling both reactive and proactive functions. The periodicity and threshold values are changed by APTEEN which is a hybrid protocol \& are used in the TEEN protocol as per the user needs \& type of the application [12]. APTEEN incorporated query handling. The following parameters are being broadcasted by the cluster heads in the APTEEN periodically.

1. ATTRIBUTE: - A set of physical parameters which is obtained by the user at his self interest.

2. THRESHOLDS: - Soft threshold (ST) and hard threshold (HT) are the two parameters of the threshold.

3. SCHEDULE: - This is a TDMA schedule, which assigns slots to each node.

4. COUNT TIME (CT):- This is the maximum time period obtained between two successive reports which is being sent by a node [12].

The environment will be sensed continuously by the nodes and only those nodes which sense a data value at or beyond the hard threshold will transmit. Once a node is able to sense a value beyond HT, it will transmit data only if the values of that attribute change by an amount that is equal to or greater than ST. If in some case a node does not send data for a particular time period which is equal to count time, it is forced to sense and retransmit the data. A TDMA schedule is used and each and every node in the cluster is assigned to a transmission slot.

Hence APTEEN will use a modified TDMA schedule for implementing the hybrid network. The main features of the APTEEN scheme will contain the following:- It will combine both the proactive and reactive policies. It will also offer a lot of flexibility by allowing the user to set the count-time interval (CT) and the threshold values for the energy consumption can be controlled by changing the count time as well as the threshold values. The actual problem involved in using the scheme is that it requires an additional complexity to implement the threshold functions and the count time. The simulation of TEEN and APTEEN had shows that the two protocols had outperformed LEACH. The APTEENs performance is somewhere between LEACH and TEEN in terms of energy dissipation and network lifetime. As it decreases the number of transmissions, TEEN always gives the best performance. The actual drawback of the two approaches are the overhead and the complexity which will associate with forming clusters at multiple levels, the method of implementing threshold based functions and the way to deal with attribute-based naming of queries.

\section{PROPOSED SK ALGORITHM}

The approach of hierarchy based routing algorithm targets to conserve energy of the sensor networks while clustering and reducing the number of hops for data transmission between clusters. In cluster formation energy is the most significant parameter. Let the initial energy of each node be considered as a constant of 8 joules. In this algorithm, every cluster has three cluster heads and 70 nodes which are divided into two groups. Each group consist of a sub-cluster head $(\mathrm{CH})$ with 35 nodes. Thus a cluster is designed to have 2 sub cluster head and a master section head. By gathering the data, Sub-cluster head check for event occurrence. If an event occurred then sub-cluster head transmit the event occurred data to the master section head. The master section head then transmits the data to the sink by multi-hop. More sensor nodes are used in a single cluster in a predefined manner. 


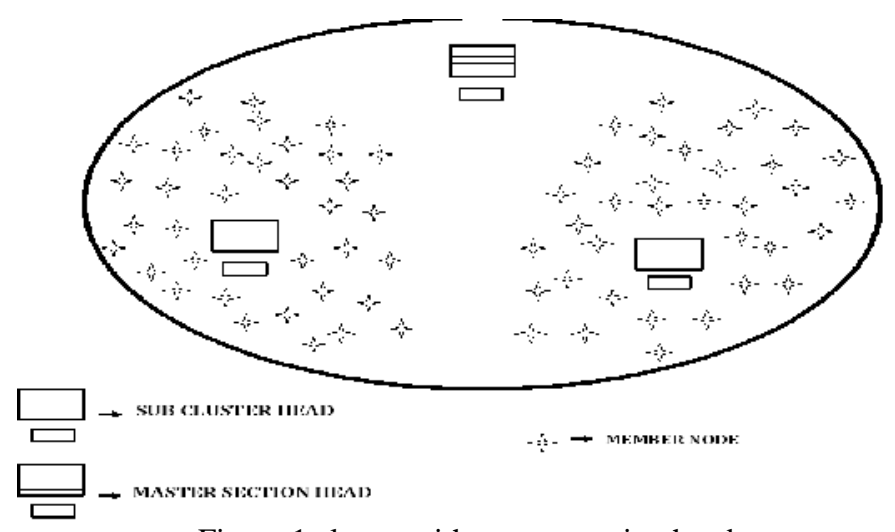

Figure 1 cluster with master section head

The energy of the network is calculated after the cluster formation, size of data packet transmitted, and number of hops to reach the destination. All of them reflect the energy consumed. Energy consumption is varied depending upon the hops and the amount of data transmitted. Message communications is the number of communications occurred between any pair of nodes while clustering the network. Hence in this proposed work cluster (as portrayed in figure 1) consists of two cluster heads as Sub cluster heads and a master section head. The data collected from the head is transmitted via a mobile agent. The mobile agent is created and passed to all the cluster heads in the sink. The agent traverses to all the clusters sequentially in a serial fashion. Each cluster contains a container, where the computation is carried out. The cluster head collects the event driven data from the environment and computes the data. The computation results are stored in the agent and the agent moves to the next cluster to collect its data. The agent transfers the data to sink after it reaches the sink finally. After receiving the entire event driven data, the sink destroys the agent. In the previous methodology of data transmission from the cluster head to the sink takes place based on the amount of data, distance, number of hops and time.

Minimum amount of data transmission is from $\mathrm{CH}$ to sink is around $60 \mathrm{mw}$ with the duration of $25 \mathrm{~ms}$. Proposed SK algorithm the mobile agent plays a role of data gathering with Zero loss of energy from the cluster heads. Event driven data are out of 100 packets approximately 5 to 10 packets, only those packets alone will be computed and transmitted by MA to the sink.

Table 1.Simulation parameters

\begin{tabular}{|l|l|}
\hline Parameter & Value \\
\hline Node Deployment region & $1000 \mathrm{~m} \mathrm{X} \mathrm{1000m}$ \\
\hline Total number of clusters & 3 \\
\hline Number of nodes in a cluster & 70 \\
\hline Initial energy of the node & 8 Joules. \\
\hline $\begin{array}{l}\text { Energy used to transmit / receive the Data( } \\
\mathrm{E}_{\text {Elec }}\end{array}$ & $50 \mathrm{~nJ} / \mathrm{bit}$ \\
\hline Energy used by the amplifier $\left(\mathrm{E}_{\mathrm{Amp}}\right)$ & $100 \mathrm{pJ} / \mathrm{bit}$ \\
\hline Data reception rate & $87.23 \%$ \\
\hline Sub Head election takes place & $\begin{array}{l}\text { When the residual energy of the sub head is } \\
\text { half of the Initial energy }\end{array}$ \\
\hline
\end{tabular}


International Journal Of Advanced Smart Sensor Network Systems (IJASSN), Vol 3, No.2, April 2013

$$
\begin{aligned}
& E_{T x}=\left(E_{\text {Elec }} * k\right)+\left(E_{\text {Amp }} * k * d^{2}\right) \\
& E_{R x}=\left(E_{\text {Elec }} * k\right)
\end{aligned}
$$

Amount of energy consumed for transmission and reception is shown in equation 4 and 5 . Where $\mathrm{k}$ is packet size of the data which is to be transmitted to a distance of $\mathrm{d}, \mathrm{E}_{\mathrm{Elec}}$ is energy spent by the transmitter or receiver device, $\mathrm{E}_{\mathrm{Amp}}$ is the energy spent for transmitter amplifier and $\mathrm{k}$ is the number of bits transmitted.

$$
\text { Residual Energy = Total energy }-(\text { Energy consumption to transmit a data }) \text {. }
$$

Energy consumed for the data that is transmitted is calculated for 50 bytes of data. In this proposed work, computation for the cluster head and cluster formation has been reduced. In the existing methodology, for each and every round the selected cluster head has to inform every member node, that it is the cluster head by calculating the residual energy of all the nodes. Here, in this algorithm, the cluster head selection takes places only when the cluster head loses its residual energy below half of the initial energy level of $\mathrm{CH}$. Thereby the complexity of cluster head selection is much reduced. Energy consumption is varied depending upon the hops and the amount of data transmitted. The deployment of more nodes is deployed in an area which will also increase the sensing capability of the network. As each cluster consists of more sensing nodes, number of clusters is reduced in this algorithm. Communication cost will also be decreased because more nodes are used in moderate areas.

\section{SIMULATION RESULTS}

Network simulator (NS2) is used for the simulation of the proposed algorithm. NS2 is an event simulator that consists of a package of tools that simulates behaviour of networks. It helps creating network topologies, analyse and log events to understand the network behaviour. According to the order of their scheduled occurrences events are queued and processed.

Each cluster consists of 70 nodes and three heads. Nodes are distributed with two groups and each groups with sub heads. Energy consumption of 50 byte of data transmission is considered and compared with the Leach protocol shown in Figure 2. For SK protocol the energy is efficient.

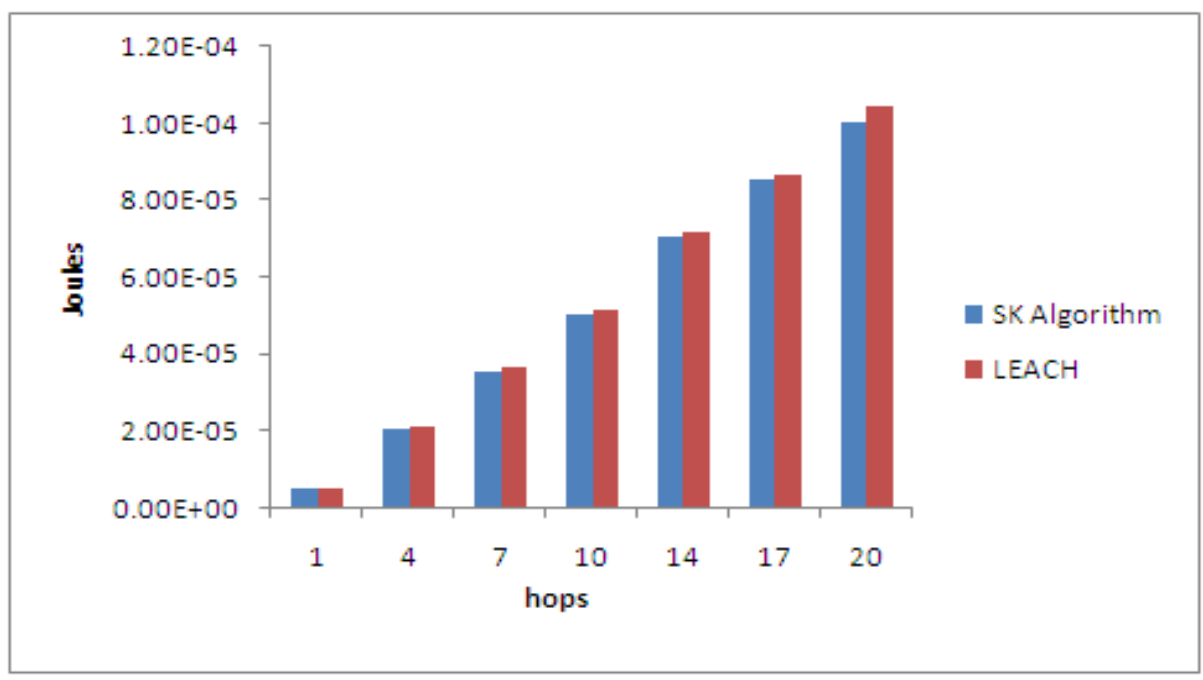

Figure 2.Energy consumption of nodes in different Hops 
Here 50 bytes of data is transmitted for a single hop. The energy calculation is only based on the data transmitted and it doesn't include the energy consumption for routing setup phase. X-axis represents the number of hops and Y-axis represents the energy consumption in joules. It shows the energy consumption details for different hops with a constant data of 50 bytes.

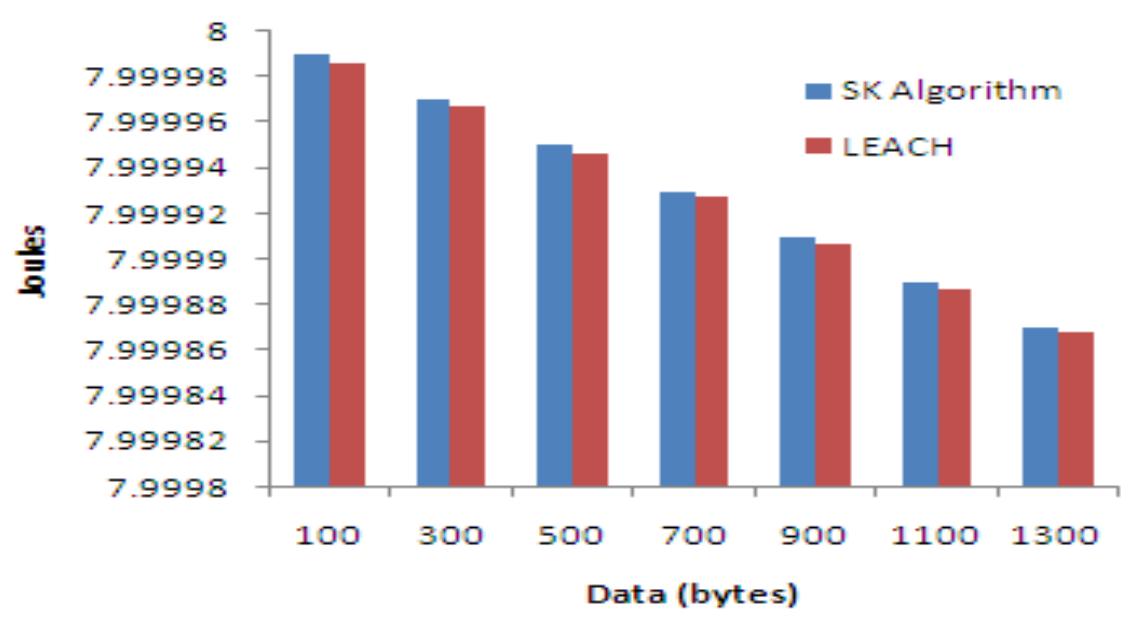

Figure 3.Residual Energy Vs packet Transmitted with in a cluster

The figure 3 shows, the decrease in the residual energy of the sensor nodes as more packets are received compared with Leach. The $\mathrm{X}$-axis represents the number of data packets and $\mathrm{Y}$-axis represents the energy consumption in joules. As more and more data packets are received, the residual energy of the nodes reduces.

The data packet which is sensed by node is passed to head, which in turn transmits to the base station. This is considered in residual energy simulation in lalgorithm is shown in Figure 4.

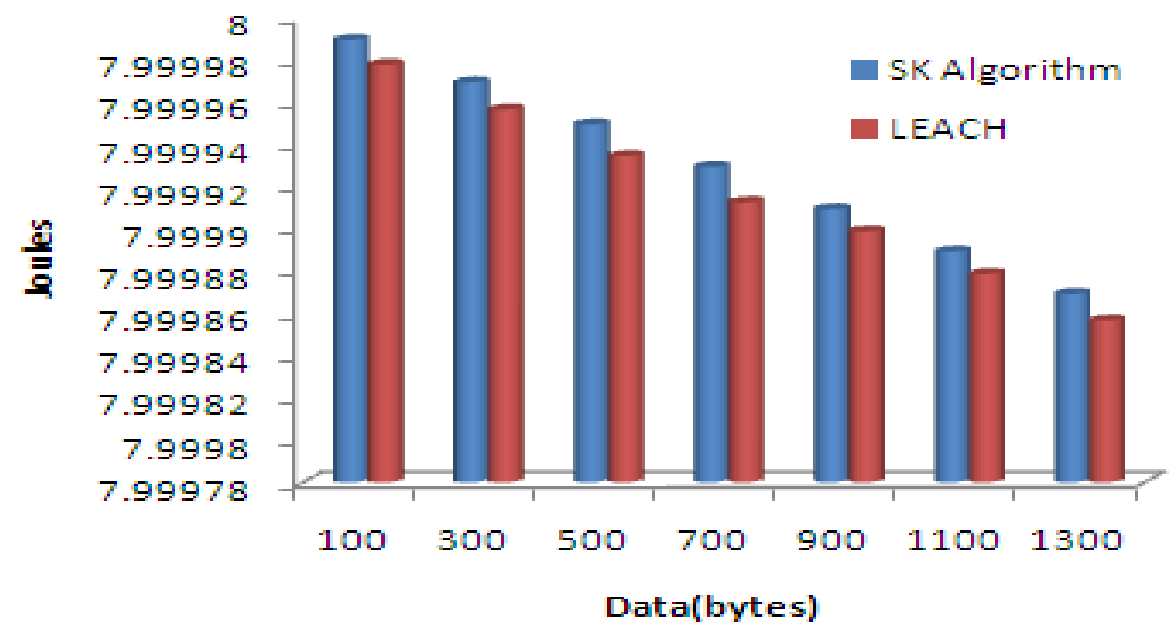

Figure 4.Residual Energy Vs Data packet Transmitted to the BS

Each group consist of 35 nodes. Here the energy consumption of each group is shown. As the network size increase the corresponding energy consumption for 500 bytes of data transmission also increases. This is illustrated in figure 5. The X-axis represents number of nodes and the $\mathrm{y}$ - 
axis represents the energy consumption in joules. The energy consumption for the data transmission increases linearly with the size of the network. If the distance between the nodes increases, the energy consumption is negligible increases.

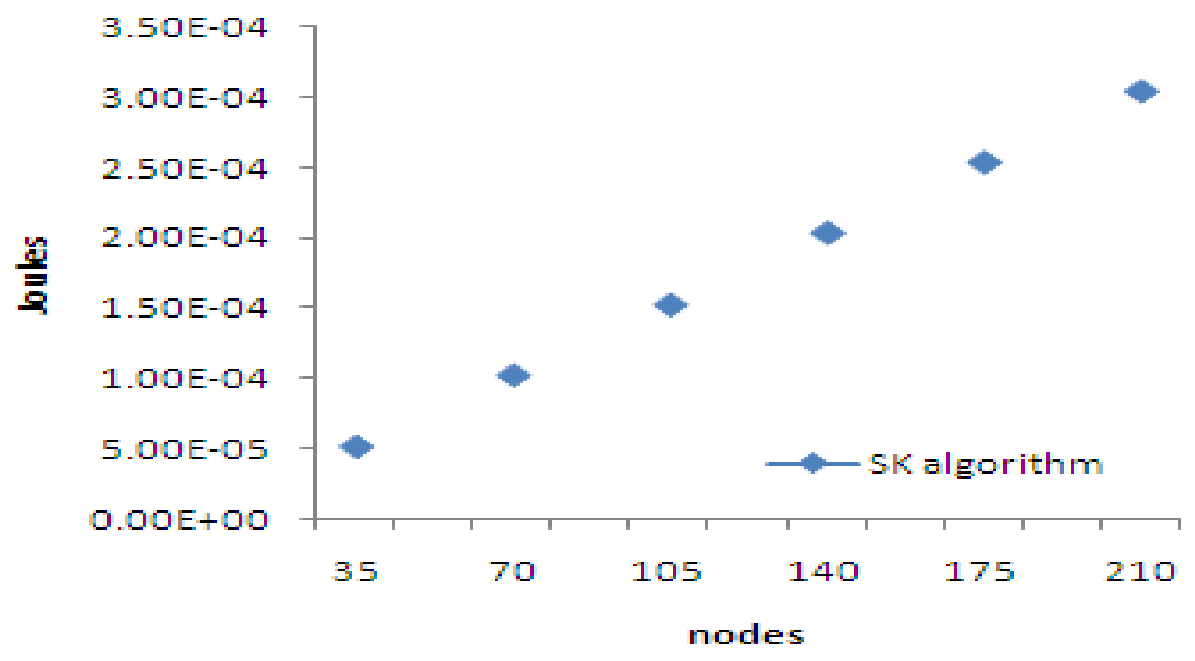

Figure 5. Residual Energy Vs nodes for 500 bytes of data transmission.

The increase in the network size, results in an increase in the number of hops for transmission of data as shown in figure 6 . The $\mathrm{X}$-axis represents the network size and the $\mathrm{Y}$-axis represents the number of hops depending on the timing. The increase in the network size will have a linear impact in the number of hops of data transmission.

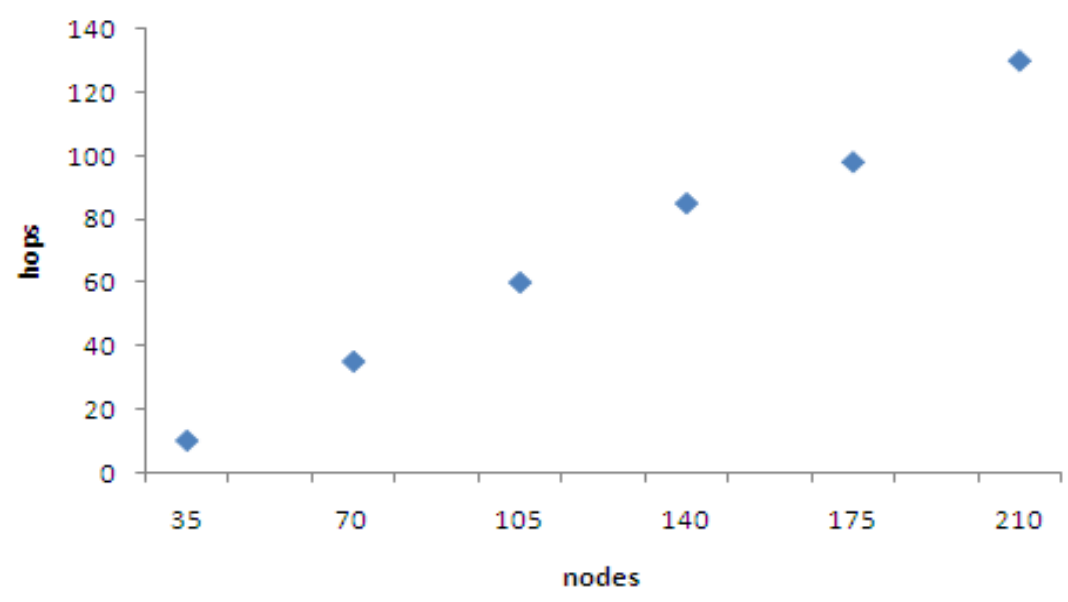

Figure 6.Network size VS Transmission Hops

The proposed SK algorithm is simulated using JADE. JADE is a software development framework which supports graphical tools and act as a middle-ware. JAVA language is used for implementation. JAVA is an object oriented programming language. Mobile agent is created in the sink, passed to heads to collect the event occurred data. Then the agent computes and transmit it to sink in tabulation is shown in figure7. 
International Journal Of Advanced Smart Sensor Network Systems (IJASSN), Vol 3, No.2, April 2013

\begin{tabular}{|c|c|c|}
\hline DSN Event occured nodes Table & & $-\square x$ \\
\hline Cluster Name & Node ID & Event Occured ... \\
\hline SubHead1 & 4 & $2012-10-14.10 .43 .28 .0$ \\
\hline SubHead1 & 15 & $2012-10-14.10 .43 .28 .0$ \\
\hline SubHead1 & 49 & $2012-10-14.10 .43 .31 .0$ \\
\hline SubHead1 & 47 & $2012-10-14.10 .43 .31 .0$ \\
\hline SubHead2 & 16 & $2012-10-14.10 .44 .45 .0$ \\
\hline SubHead2 & 12 & $2012-10-14.10 .44 .47 .0$ \\
\hline SubHead2 & 47 & $2012-10-14.10 .44 .52 .0$ \\
\hline SubHead3 & 11 & $2012-10-14.10 .46 .8 .0$ \\
\hline SubHead3 & 4 & $2012-10-14.10 .46 .10 .0$ \\
\hline SubHead3 & 17 & $2012-10-14.10 .46 .15 .0$ \\
\hline SubHead3 & 7 & $2012-10-14.10 .46 .18 .0$ \\
\hline SubHead3 & 48 & $2012-10-14.10 .46 .19 .0$ \\
\hline
\end{tabular}

Figure7. Event occurred data is tabulated in sink

\section{CONCLUSION}

The energy efficient hierarchy based protocols which are proposed in this paper have resulted in a significant improvement in the performance of the network over a centralised approach. As a result of the algorithm proposed, where the initial energy of the nodes and cluster head are defined, the energy consumed for the computation is less as shown in the simulation results. Based on the cluster head selection proposed in this paper, the complexity of the network and its energy consumption is much reduced. The nodes being deployed in large numbers within moderate area, demonstrates the reduction in communication cost. This algorithm is extended to use mobile agent for data transmission, which facilitates data gathering and data transmission in an efficient manner. This reduces the complexity and the Latency.

\section{REFERENCE}

[1] Y. Xu, J. Heinemann and D. Estrin. “Geography-informed energy conservation for ad hoc routing”, In Proceedings of the Seventh Annual International Conference on Mobile Computing and Networking (MobiCom), 2001.

[2] Boulis A, Ganeriwal S and Srivastava M B,“ Aggregation in sensor networks: an Energy-Accuracy Trade-Off” ,Elsevier journal of Ad Hoc Networks, 2003, 1(1), pp.317-331.

[3] C. Intanagonwiwat, R. Govindan andEstrin D. Directed diffusion: A scalable and robustcommunication paradigm for sensor networks. In Proceedings of theSixth Annual InternationalConference on Mobile Computing and Networks (MobiCom), 2000.

[4] Abdul Sattar Malik, Jingmingkuang,Jiakang Liu, Wang Chong, "Energy Consumption \& Lifetime Analysis in Cluster-based Wireless Sensor Networks for Periodic monitoring Applications",International conference on Network security, wireless Communications and trusted computing, IEEE November2009, pp:657-661.

[5] KeyhanKhamforoosh, Hana Khamforoush, "A New Routing Algorithm for Energy Reduction in Wireless sensor Networks", IEEE conference 2009, pp: 505-509.

[6] Mohammad Ilyas and ImadMahgoub, "Handbook of Sensor Networks: Compact Wireless and Wired sensing systems" by CRC press.

[7] M.J.Handy, M.Haase, D. Timmernann, "Low Energy Adaptive Clustering Hierarchy with Deterministic Cluster-Head Selection”, International conference on University of Rostock ,2004, PP:211-216. 
International Journal Of Advanced Smart Sensor Network Systems (IJASSN), Vol 3, No.2, April 2013

[8] sudakshinadasgupta, paramarthadutta,"An improved LEACH approach for head selection stratergy in a fuzzy-c means induced clustering of a wireless sensor network" IEMCON 2011 organised by IEM to collaboration with IEEE on 5th and 6th of jan 2012,PP-203-208.

[9] T. Rappaport, Wireless Communications:Principles\&Practice.EnglewoodCliffs,NJ: Prentice- Hall, 1996.

[10] J. Elson and D. Estrin, “An Address-Free Architecture for Dynamic Sensor Networks". Technical Report 00-724, Computer Science Department, USC, January 2000.

[11] DionisisKandris, PanagiotisTsioumas, Anthony Tzes, George Nikolakopoulos and Dimitrios D. Vergados, "Power conservation through Energy Efficient Routing in Wireless sensor Networks", sensor journal, September 2009, PP: 7320-7342.

[12] W. B. Heinzelman. "Application-Specific Protocol Architectures for Wireless Networks".PhD thesis, Massachusetts Institute of Technology, June 2000. 